\title{
The Clinician Scientist
}

\author{
David R Williamson, Salmaan Kanji, and Lisa Burry
}

Can J Hosp Pharm. 2021;74(2):130-4

\section{INTRODUCTION}

Clinical research is an essential component of health sciences that focuses on improving knowledge about the diagnosis, prevention, and treatment of diseases, through a variety of methodologies, with the ultimate intent of enhancing the health and quality of life of individual patients or populations. ${ }^{1-3}$ Because health problems are multidimensional, an interdisciplinary approach to research is necessary to establish innovative and adaptive solutions. ${ }^{4-6}$ Clinician scientists lead clinical research, are essential components of the research infrastructure of academic health care centres, and have traditionally had a background in medicine. ${ }^{3}$ However, clinicians from other health care sciences, such as nutrition, nursing, physiotherapy, and pharmacy, have become increasingly common in the past 20 years. $^{7}$ The unique competencies and perspectives of pharmacists make them suitable to lead multidisciplinary research teams to address unanswered therapy-related questions and to close important gaps between drug-related knowledge and the bedside. ${ }^{3}$ Although we are not aware of official data, it is our observation that clinician-scientist pharmacists are increasingly securing funding from federal funding agencies (e.g., Canadian Institutes of Health Research [CIHR] or the US National Institutes of Health) in recent years, either as members of interdisciplinary research teams or as principal investigators. ${ }^{5,8}$ In addition, pharmacists are increasingly publishing in high-impact medical journals (including the New England Journal of Medicine, The Lancet, BMJ, JAMA, Chest, Cochrane Database of Systematic Reviews, and CMAJ). ${ }^{9-20}$ In 2010, an American College of Clinical Pharmacy (ACCP) committee on the pharmacist as clinician scientist searched the ClinicalTrials.gov trial registration website using the term "PharmD" and found a total of 523 active studies with pharmacists as principal investigators. ${ }^{8}$ On November 16, 2020, we repeated the ACCP's search and found 2094 studies, an important increase in the registration of studies by pharmacists in just 10 years. Pharmacists have also been recognized as principal investigators by granting agencies. In fact, it was nearly 40 years ago, in 1983, that the ACCP obtained clarification that pharmacists were allowed by the US Food and Drug Administration to be principal investigators. ${ }^{8}$
Clinician-scientist pharmacists are typically specialists in a specific clinical area who divide their time between practice and research. Relevant research ideas are drawn from their pharmacy practice experiences and generate new knowledge that can be directly applied in the clinical setting to improve patient outcomes. ${ }^{7}$ Traditionally, pharmacy-based researchers have had an academic background, focusing on specific disciplines such as pharmacokinetics, pharmacology, therapeutics, pharmacogenomics, drug safety, pharmacoepidemiology, and pharmacoeconomics. Our own observations indicate that an increasing number of pharmacy clinicians have become involved in clinical research in various areas, including cardiology, critical care, geriatrics, infectious diseases, mental health, nephrology, and oncology. Despite the growing body of new knowledge that results directly from pharmacist-initiated research, we believe that pharmacists remain underrepresented among clinician scientists. A Canadian survey published in 2012 indicated that pharmacists were interested in becoming more involved in clinical research; however, respondents noted key barriers that needed to be addressed, such as lack of time, lack of institutional support, and lack of expertise or training. ${ }^{21}$

The objective of the current review is threefold: to describe the suggested training and role of the clinician-scientist pharmacist, to highlight barriers and facilitators to the training and employment of pharmacists to assume this role, and to identify keys for a successful career as a clinician scientist.

\section{TRAINING}

There is no single specific pathway for pharmacists to pursue a career as clinician scientists. ${ }^{2}$ Following an entry-level baccalaureate or doctoral degree in pharmacy, many clinician scientists will pursue training with a year 1 residency program or a master's degree in advanced pharmacotherapy and possibly a year 2 residency. Although these programs generally seek to develop some research competencies, the curriculums are structured to ensure that individuals can become clinical experts, with only a limited introduction to research. As such, advanced training in research methodology and statistics is necessary if a pharmacist is to become an independent, competitive researcher and be successful in obtaining extramural funding. ${ }^{22}$ 
A variety of program options are available to pharmacists for advanced research training, which can be classified into 2 main pathways: fellowships and graduate studies. Mostly available in the United States, fellowships are 1- or 2-year hospital- or industry-based programs. Fellowship programs are typically affiliated with a university and focus on various methods of research in a wide variety of clinical domains. At the time of writing (late 2020), the ACCP listed more than 60 fellowship programs that were available in the United States. Fellowship programs vary considerably with respect to clinical subspecialties (e.g., infectious diseases, transplant medicine, critical care), research methodologies (e.g., clinical research, pharmacokinetics, health services research, laboratory-based research), and settings (e.g., university laboratory, hospital and community settings, pharmaceutical industry). ${ }^{23}$ Most fellowship programs involve some aspect of patient care and academia; however, $50 \%$ to $75 \%$ of a fellow's time is dedicated to research activities. The objectives, expectations, and end goals of these programs vary widely, to provide a highly individualized experience to the trainee. Fellowship programs seeking endorsement by the ACCP must meet specific minimum criteria, including at least 3000 hours dedicated to research activities over 2 years. ${ }^{23}$ Eligible applicants typically are required to have a master's or doctoral degree (PharmD) and residency training or equivalent clinical experience. Trainees in research fellowships can expect to gain experience in all aspects of clinical research, including protocol development, grantsmanship, study design, study coordination and conduct, statistical analysis, and manuscript preparation. ${ }^{24}$

Graduate studies, including master's (MSc) and doctoral (PhD) programs, are offered by various health science faculties, including faculties of pharmacy, nursing, and medicine. Pharmacy faculties across Canada and the United States offer $\mathrm{MSc}$ and $\mathrm{PhD}$ programs in pharmaceutical sciences, with various options such as clinical sciences and translational research. MSc degrees generally take 2 years to complete, whereas $\mathrm{PhD}$ programs require 4 to 6 years. MSc programs involve coursework in research methods and statistics, a research project leading to the publication of 1 or 2 scientific articles, and preparation of a thesis. $\mathrm{PhD}$ programs generally include additional research methods and advanced statistics courses, the development of a research program including protocol writing and grantsmanship, the publication of 3 or more scientific articles, a qualifying examination, and the writing of a thesis. Dual-degree PharmD/MSc or PharmD/ $\mathrm{PhD}$ programs, which are becoming more prevalent in the United States, have the advantage of streamlining research training and providing students with a clear path toward graduate studies. Although similar programs for physicians (MD/MSc and $\mathrm{MD} / \mathrm{PhD}$ programs) are increasingly popular among faculties of medicine and medical students in Canada, Canadian faculties of pharmacy have yet to offer such programs. Postdoctoral studies, which follow graduate studies, are highly encouraged for pharmacists who intend to pursue research and academia as their primary career, but may also enable clinician scientists to acquire additional experience and competencies, thereby increasing their competitiveness in obtaining research funding. In Canada, many clinician-scientist pharmacists have pursued $\mathrm{MSc}$ or $\mathrm{PhD}$ programs in clinical epidemiology, experimental medicine, and clinical sciences offered in faculties of medicine.

Both training paths offer advantages, but they also have drawbacks, which have become a source of long-standing debate in the United States. ${ }^{22,23,25}$ Fellowship programs offer less academic classwork than graduate studies, along with a salary, a more hands-on approach to research, and the potential to conduct and participate in multiple research projects rather than just thesis work; however, no such programs are offered in Canada. In contrast, graduate studies offer an opportunity to acquire a strong theoretical foundation in research methods, a structured academic program, and a recognized degree that can open the door to academia, but they are long and arduous endeavours that may depend on grants for financial support, although certain faculties offer some forms of funding (e.g., internal grants or teaching assistant positions). Most importantly, both fellowships and graduate studies enable the development of essential skills, such as critical reasoning, problem-solving, and hypothesis generation. In addition, these programs facilitate the development of a research network.

In both fellowship programs and graduate studies, the most important elements to consider are the choice of the preceptor/professor and the institution. Finding the right fit with a supervisor and institution will not only provide the best opportunity to ensure development of targeted research competencies through the proposed research program, but will also provide opportunities to develop research networks and mentoring relationships.

\section{CURRENT STATE}

Historically, physicians have made up the majority of clinician scientists, and this concept is well established in the medical field in Canada, with a number of structured programs and career paths available once medical training is complete. ${ }^{26,27}$ For other health care professionals, the development of a career path for clinician scientists is less well established. ${ }^{7}$ Although the exact number of clinician-scientist pharmacists in Canada is not known, it is likely small relative to the pharmacy workforce as a whole and relative to the clinician scientists from other health care professions (e.g., nursing and medicine). In Canada, most clinician-scientist pharmacists practise in academic health centres that have an affiliation with a hospital research centre, and they have university cross-appointments as either clinical professors or clinician scientists. Some faculties, such as the Leslie Dan Faculty of Pharmacy at the University of Toronto, have developed 
specific career paths for clinician scientists. These relationships and affiliations are necessary for all clinician scientists to enable and support research endeavours by making available research infrastructure, research networks, and resources such as methodological, contract, and financial support. In some cases, the clinician scientist will obtain career grants from granting agencies (federal or provincial) or local health science centres. For example, in the province of Quebec, the Fonds de recherche Santé-Québec offers clinician scientist grants for health care professionals. These 4-year grants are competitive, cover about half of a person's salary, and are renewable to a maximum of 3 terms (Junior 1, Junior 2, and Senior Clinician Scientist). Applicants must have a faculty appointment and must guarantee that at least $50 \%$ of their time is devoted to their research program. The CIHR offers a similar program. To our knowledge, however, few clinicianscientist pharmacists have followed this path, leaving these opportunities to other health care professionals.

The profession of pharmacy needs to engage and build a workforce of clinician-scientist pharmacists in Canada. At the present time, we believe that clinician-scientist pharmacists are rare in hospital research centres and pharmacy faculties, with most pharmacists having to choose between full-time pharmacy practice and full-time research. Ideally, we should strive as a profession to have at least one trained clinicianscientist pharmacist within the ranks of every universityaffiliated hospital pharmacy department, to develop and support programs of research and to mentor or supervise research trainees. For this to happen, there is a need to develop university cross-appointed and funded clinicianscientist positions within hospital pharmacy departments, with $50 \%$ or more protected research time. From the perspective of the pharmacy department there are benefits to this investment. Many hospitals and their pharmacy departments identify research as a priority within their vision and mission statements. Investing in clinician scientists, including clinician-scientist pharmacists, is one way to support these objectives. Research productivity can provide regional, national, and international recognition, which can be used in the recruitment of staff and learners. The designated clinician-scientist pharmacist could provide methodological support and mentorship to residents conducting research projects, as well as to other clinical staff with less experience, with the overall goal of promoting an integrative culture between clinical work and research. Furthermore, in large institutions, the pharmacy department is often involved in the conduct of many trials and research projects initiated by other researchers, clinicians, and industry partners. Having a pharmacy research leader within the department, someone who plays a supportive role in the conduct of research involving the pharmacy, could ensure that the department's needs and role are always considered and that pharmacy is represented within affiliated research organizations and research ethics boards.

\section{BARRIERS AND FACILITATORS}

Over recent years, there has been a reduction in the number of clinical research trainees across all health professions. ${ }^{27,28}$ One qualitative study identified 3 main barriers perceived by clinical scientists in Canada: research training, research salaries, and research grants. ${ }^{29}$ To these, we must add the challenges of having an active clinical practice and maintaining up-to-date clinical competencies, while balancing the workload associated with staying competitive in multidisciplinary research funding opportunities (e.g., through CIHR), managing active research projects, and disseminating their results. Lack of support early in a person's career, particularly among those in their first academic position, has also been identified as a key barrier to maintaining a clinician-scientist workforce. ${ }^{27}$

Maintaining an active clinical practice represents an additional challenge for clinician scientists who are trying to secure research funds. An applicant's research experience is an important criterion in these efforts, and clinician scientists have the added difficulty of competing with fulltime researchers. ${ }^{7,29}$ Lack of dedicated time for research is not unique to pharmacists or clinician scientists. Protected research time is more likely in academic positions, but in both academic and clinical settings it is usually dependent on grant funding. Some grants and funding awards will even allow for salary support, which typically is used to fund the protected research time, but this model depends on the duration of funding and means that sustainability is necessarily competitive.

For pharmacists who are willing to develop a clinical research career, specific research training opportunities are scarce. There is a need to develop dedicated programs within Canadian faculties of pharmacy and thus a critical need for clinician-scientist pharmacists to act as mentors and to seek positions as professors and research directors. Pharmacy faculties should aim to develop flexible programs for practising clinical pharmacists who wish develop research competencies, as well as combined PharmD/MSc or PharmD/ $\mathrm{PhD}$ programs in clinical research to recruit young students early in their studies and new clinician-scientist positions to enable the training of graduate student pharmacists. Strategies for more recruitment of pharmacy students to graduate programs also need to be introduced. These strategies may already exist in the basic science departments within pharmacy faculties and could be adopted by pharmacy practice departments.

To advance clinical pharmacy research, pharmacy students need to be more aware of research career paths early in their programs of study. Enabling undergraduate pharmacy students to participate in research opportunities early on is one of the keys to success. ${ }^{30}$ During first-year residency programs, research projects should be supported by experienced research teams to ensure that research experiences 
are positive and to ensure that residents have opportunities to publish their work. ${ }^{31}$ Given that most residency projects in Canadian and US programs remain unpublished, ${ }^{32}$ there is clear room for improvement. In addition, pharmacy education needs to engender an attitude of inquisitiveness and scholarly thinking among pharmacists. ${ }^{33}$ This attitude should be nurtured early in the pharmacy curriculum (or even before) and groomed throughout entry-level studies to ensure the training of candidates who will engage in advanced research training and provide a pipeline of clinician scientists. Students should be encouraged to move beyond studying with the basic goal of passing exams and to focus more on developing skills in critical thinking, problem-solving, and scientific reasoning. Suggestions to improve critical thinking have included enabling a thoughtful learning environment, enabling students to understand the processes needed to execute cognitive operations, and guiding and supporting their efforts until they can perform on their own. ${ }^{33}$

\section{FUTURE DIRECTIONS}

The funding of dedicated research positions for clinicianscientist pharmacists by health care institutions, pharmacy faculties, granting agencies, or a combination of these is essential for the career development of clinician-scientist pharmacists. Canada has a highly competitive research environment, with more good research projects being proposed than funding opportunities can support, which leads to low success rates in the major grant competitions (e.g., those of the CIHR). ${ }^{34}$ Clinician-scientist pharmacists should also explore alternative granting sources such as foundations and other local granting agencies.

A recent consensus panel for the training and earlycareer support of physician scientists issued recommendations that could inspire the pharmacy profession. ${ }^{27}$ The panel recommended the establishment of a national council to provide oversight of clinician-scientist programs, capacity development for funding and mentorship support, and development of interdisciplinary networks of clinicianscientists, and to ensure that faculties cover the scientific basis of health care and include research methods in their curriculums. ${ }^{27}$ In particular, the development of mentorship programs within the pharmacy profession seems essential to support clinician scientists and to enable them to lead successful research and clinical careers. ${ }^{3}$ Identifying and training mentors should thus be a priority within our profession.

Finally, given that initiating a research program is not an easy undertaking and necessitates plenty of hard work and passion, aspiring clinician-scientist pharmacists need to be dedicated and should expect to work extremely hard, often on their own time, to obtain grants, design studies, and be successful in research. Establishing a record of success in a research program is one potential way of negotiating and obtaining protected research time for future projects.

\section{CONCLUSION}

Clinician scientists represent an essential component of the research infrastructure of academic health care centres, but few pharmacists have followed this career path. Although the pharmacy profession has made important strides over the past decades, Canada needs to engage and build a workforce of pharmacists that includes clinician scientists. In the past 3 to 4 decades, pharmacists have established themselves as essential members of the bedside clinical team. They should now use their drug-related knowledge to inform clinical research and patient care.

\section{REFERENCES}

1. Westfall JM, Mold J, Fagnan L. Practice-based research—"Blue Highways" on the NIH roadmap. JAMA. 2007;297(4):403-6.

2. Dowling TC, Murphy JE, Kalus JS, Nkansah NT, Chappell JS, Wiederhold NP, et al.; American College of Clinical Pharmacy. Recommended education for pharmacists as competitive clinical scientists. Pharmacotherapy. 2009;29(2):236-44.

3. Bookey-Bassett S, Bianchi A, Richards J, Kelly H. Overcoming challenges to support clincian-scientist roles in Canadian academic health sciences centres. Healthc Q. 2019;22(1):60-6.

4. Hall JG, Bainbridge L, Buchan A, Cribb A, Drummond J, Gyles C, et al. A meeting of minds: interdisciplinary research in the health sciences in Canada. CMAJ. 2006;175(7):763-71.

5. Dolovich L. Playing in the sandbox: considerations when leading or participating on a multidisciplinary research team. Can J Hosp Pharm. 2015;68(5):401-5.

6. Nair KM, Dolovich L, Brazil K, Raina P. It's all about relationships: a qualitative study of health researchers' perspectives of conducting interdisciplinary health research. BMC Health Serv Res. 2008;8: Article 110.

7. MacDonald SE, Sharpe HM, Shikako-Thomas K, Larsen B, MacKay L. Perspective: Entering uncharted waters: navigating the transition from trainee to career for the nonphysician clinician-scientist. Acad Med. 2013;88(1):61-6.

8. Burton ME, Munger MA, Bednarczyk EM, Davis LE, Davis GA, Elliott ME, et al. Update: the clinical pharmacist as principal investigator. Pharmacotherapy. 2010;30(12):485e-489e.

9. Dolovich L, Oliver D, Lamarche L, Thabane L, Valaitis R, Agarwal G, et al. Combining volunteers and primary care teamwork to support health goals and needs of older adults: a pragmatic randomized controlled trial. CMAJ. 2019;191(18):E491-E500.

10. Victor RG, Lynch K, Li N, Blyler C, Muhammed E, Handler J, et al. A cluster-randomized trial of blood-pressure reduction in black barbershops. N Engl J Med. 2018;378(14):1291-301.

11. Mehta S, Burry L, Cook D, Fergusson D, Steinberg M, Granton J, et al.; SLEAP Investigators; Canadian Critical Care Trials Group. Daily sedation interruption in mechanically ventilated critically ill patients cared for with a sedation protocol: a randomized controlled trial. JAMA. 2012;308(19):1985-92.

12. Sobieraj DM, Baker WL, Nguyen E, Weeda ER, Coleman CI, White $\mathrm{CM}$, et al. Association of inhaled corticosteroids and long-acting muscarinic antagonists with asthma control in patients with uncontrolled, persistent asthma: a systematic review and meta-analysis. JAMA. 2018 319(14):1473-84.

13. Qato DM, Ozenberger K, Olfson M. Prevalence of prescription medications with depression as a potential adverse effect among adults in the United States. JAMA. 2018;319(22):2289-98.

14. Carter AA, Gomes T, Camacho X, Juurlink DN, Shah BR, Mamdani MM. Risk of incident diabetes among patients treated with statins: population based study. BMJ. 2013;346:f2610. 
15. Bress AP, Bellows BK, King JB, Hess R, Beddhu S, Zhange Z, et al.; SPRINT Research Group. Cost-effectiveness of intensive versus standard blood-pressure control. N Engl J Med. 2017;377(8):745-55.

16. Devlin JW, Skrobik Y, Gelinas C, Needham DM, Slooter AJC, Pandharipande $\mathrm{PP}$, et al. Clinical practice guidelines for the prevention and management of pain, agitation/sedation, delirium, immobility, and sleep disruption in adult patients in the ICU. Crit Care Med. 2018;46(9): e825-e873.

17. Burry L, Hutton B, Williamson DR, Mehta S, Adhikari NKJ, Cheng W, et al. Pharmacological interventions for the treatment of delirium in critically ill adults. Cochrane Database Syst Rev. 2019;9(9):CD011749.

18. Jackevicius CA, Tu JV, Demers V, Melo M, Cox J, Rinfret S, et al. Cardiovascular outcomes after a change in prescription policy for clopidogrel. N Engl J Med. 2008;359(17):1802-10.

19. Burry LD, Barletta JF, Williamson D, Kanji S, Maves RC, Dichter J, et al. It takes a village...: contending with drug shortages during disasters. Chest. 2020;158(6):2414-24

20. Hong LT, Liou TG, Deka R, King JB, Stevens V, Young DC. Pharmacokinetics of continuous infusion beta-lactams in the treatment of acute pulmonary exacerbations in adult patients with cystic fibrosis. Chest. 2018;154(5):1108-14.

21. Perreault MM, Thiboutot Z, Burry LD, Rose L, Kanji S, LeBlanc JM, et al. Canadian survey of critical care pharmacists' views and involvement in clinical research. Ann Pharmacother. 2012;46(9):1167-73.

22. American College of Clinical Pharmacy Research Affairs Committee; Fagan SC, Touchette D, Smith JA, Sowinski KM, Dolovich L, Olson $\mathrm{KL}$, et al. The state of science and research in clinical pharmacy. Pharmacotherapy. 2006;26(7):1027-40.

23. Mueller EW, Bishop JR, Kanaan AO, Kiser TH, Phan H, Yang KY. Research fellowship programs as a pathway for training independent clinical pharmacy scientists. Pharmacotherapy. 2015;35(3):e13-e19.

24. Guidelines for clinical research fellowship training programs. American College of Clinical Pharmacy; 2016 Oct 1 [cited 2021 Feb 26]. Available from: https://www.accp.com/resandfel/guidelines.aspx

25. Parker RB, Ellingrod V, DiPiro JT, Bauman JL, Blouin RA, Welage LS. Preparing clinical pharmacy scientists for careers in clinical/ translational research: can we meet the challenge? ACCP Research Affairs Committee commentary. Pharmacotherapy. 2013;33(12):e337-e346.

26. Appleton CT, Belrose J, Ward MR, Young FB. Strength in numbers: growth of Canadian clinician investigator training in the 21st century. Clin Invest Med. 2013;36(4):E163-E169.

27. Strong MJ, Busing N, Goosney DL, Harris KA, Horsley T, Kuzyk A, et al. The rising challenge of training physician-scientists: recommendations from a Canadian national consensus conference. Acad Med. 2018; 93(2):172-8
28. Weggemans MM, Friesen F, Kluijtmans M, Prakken B, Ten Cate O, Woods NN, et al. Critical gaps in understanding the clinicianscientist workforce: results of an international expert meeting. Acad Med. 2019;94(10):1448-54.

29. Lander B, Hanley GE, Atkinson-Grosjean J. Clinician-scientists in Canada: barriers to career entry and progress. PLoS One. 2010;5(10):e13168.

30. Russell SH, Hancock MP, McCullough J. The pipeline. Benefits of undergraduate research experiences. Science. 2007;316(5824):548-9.

31. Williamson DR, Lalonde L. Should residency projects involve more than one resident? The "pro" side. Can J Hosp Pharm. 2014;67(1):48-9.

32. Hung M, Duffett M. Canadian pharmacy practice residents' projects: publication rates and study characteristics. Can J Hosp Pharm. 2013; 66(2):86-95.

33. Persky AM, Medina MS, Castleberry AN. Developing critical thinking skills in pharmacy students. Am J Pharm Educ. 2019;83(2):7033.

34. Science funding in Canada - statistics. Canadian Association for Neuroscience; 2020 [cited 2020 Nov 3]. Available from: https://can-acn.org/ science-funding-in-canada-statistics/

David R Williamson, BPharm, MSc, PhD, is a Full Clinical Professor with the Faculty of Pharmacy, Université de Montréal; a Clinical Scientist with the Research Centre, Centre intégré universitaire de santé et de services sociaux du Nord-de-I'Île-de-Montréal; and a Clinical Pharmacist with the Pharmacy Department, Hôpital du Sacré-Cœur de Montréal, Montréal, Quebec.

Salmaan Kanji, BScPharm, PharmD, is an Associate Scientist with the Clinical Epidemiology Program, Ottawa Hospital Research Institute, Ottawa, Ontario; a Clinical Pharmacy Specialist-Critical Care with the Department of Pharmacy, The Ottawa Hospital, Ottawa, Ontario; an Assistant Professor with the Faculty of Medicine, University of Ottawa, Ottawa, Ontario; and an Adjunct Professor with the Faculty of Pharmacy, Université de Montréal, Montréal, Quebec.

Lisa Burry, BScPharm, PharmD, FCCP, FCCM, is a Clinician Scientist with the Department of Pharmacy and Medicine, Sinai Health System, an Associate Professor, Leslie Dan Faculty of Pharmacy, University of Toronto, Toronto, Ontario, and a PhD candidate at the University of Toronto.

Competing interests: None declared.

Address correspondence to:

Dr David R Williamson

Pharmacy

Hôpital du Sacré-Coeur de Montréal

5400 , boul. Gouin 0.

Montréal QC H4J 1C5

email: david.williamson@umontreal.ca

\section{Advanced Pharmacist Practitioner Series}

The article about pharmacists as clinician scientists that appears in this issue concludes the Advanced Pharmacist Practitioner Series, which has been focused on enabling hospital pharmacists and pharmacy departments to advance and broaden the scope of pharmacy practice across Canada. The following is a complete list of the articles that have appeared in this series.

The advanced pharmacist practitioner: a new series in the Canadian Journal of Hospital Pharmacy (Stephen Shalansky). Can J Hosp Pharm. 2019;72(1):42-8.

Advanced strategies in pharmacy experiential education (Michael Legal). Can J Hosp Pharm. 2019;72(3):239-44.

Pharmacy informatics: where medication use and technology meet (Daniel Cortes, Jodie Leung, Andrea Ryl, and Jenny Lieu). Can J Hosp Pharm. 2019;72(4):320-6.
Role of the US Veterans Health Administration clinical pharmacy specialist provider: shaping the future of comprehensive medication management (M Shawn McFarland, Julie Groppi, Terri Jorgenson, Tera Moore, Heather Ourth, Andrea Searle, and Anthony Morreale). Can J Hosp Pharm. 2020;73(2):152-8.

Recognition of advanced practice pharmacists in Australia and beyond: considerations for Canadian practitioners (Rochelle M Gellatly and Kirsten Galbraith). Can J Hosp Pharm. 2020;73(3):225-31.

The clinician scientist (David R Williamson, Salmaan Kanji, and Lisa Burry). Can J Hosp Pharm. 2021;74(2):130-4. 